\title{
Philosophiques
}

\section{Utopie et liberté}

\section{Jacques D’Hondt}

Volume 1, numéro 2, octobre 1974

URI : https://id.erudit.org/iderudit/203018ar

DOI : https://doi.org/10.7202/203018ar

Aller au sommaire du numéro

Éditeur(s)

Société de philosophie du Québec

ISSN

0316-2923 (imprimé)

1492-1391 (numérique)

Découvrir la revue

Citer ce document

D’Hondt, J. (1974). Utopie et liberté. Philosophiques, 1(2), 141-156.

https://doi.org/10.7202/203018ar

Ce document est protégé par la loi sur le droit d'auteur. L'utilisation des services d'Érudit (y compris la reproduction) est assujettie à sa politique d'utilisation que vous pouvez consulter en ligne.

https://apropos.erudit.org/fr/usagers/politique-dutilisation/
Cet article est diffusé et préservé par Érudit.

Érudit est un consortium interuniversitaire sans but lucratif composé de l’Université de Montréal, l'Université Laval et l'Université du Québec à Montréal. Il a pour mission la promotion et la valorisation de la recherche. https://www.erudit.org/fr/ 


\title{
INTERVENTIONS
}

\section{UTOPIE ET LIBERTÉ}

\author{
par Jacques D'HONDT
}

On a dès longtemps soupçonné l'utopie de dissimuler sa véritable nature sous un air bohème. Il suffit d'une douce violence pour lui faire avouer des origines et une résidence.

Mais n'échange-t-on pas alors un mensonge contre une illusion? Le dernier refuge de l'utopie reste peut-être encore à découvrir.

\section{Le double jeu de l'utopie}

Elle naît d'un sentiment de servitude et d'une fièvre de liberté. Elle vise l'émancipation de l'homme sans disposer des moyens utiles, et, pour remporter tout de même une victoire, elle consent à payer un prix exorbitant: la plénitude concrète contre un mirage!

Elle rompt radicalement toutes les amarres, et d'abord l'espace, avec sa principale épissure, le temps. Comment s'assurer une plus grande liberté de manoeuvre?

Ce qui ne prétend exister nulle part ne se soumet à rien. L'absence garantit aussi la sécurité: la route des miasmes est coupée.

De cette manière ce qui est libre et sain se dispense d'exister : le rationnel n'est pas réel. L'utopie récuse les conditions premières de la présence et elle s'enchante de sa liberté négative. Parvient-elle toutefois à masquer longtemps la mauvaise foi de son procédé ? L'imagination, fière de congédier l'espace, se montre bien incapable de construire hors de lui. Ses moindres châteaux 
sont en Espagne. C'est dans des métaphores spatiales qu'elle affiche son reniement.

Quelle timidité, auprès de l'audace des religions, qui exilent la liberté dans un au-delà ! Quelle modestie, auprès de la démesure des philosophies qui, pour mieux voler, croient-elles, suppriment la résistance de l'air! Le philosophe théorise librement dans le vide. Ses abstractions liquident le temps et l'espace beaucoup plus brutalement que ne le ferait l'utopie la plus effrontée. Ses «maisons de coucous dans les nuées» conceptuelles s'affranchissent même de l'image!

Dans l'utopie, l'absence de lieu voudrait tenir, si l'on ose dire, toute la place. Elle désire la discontinuité absolue, sans grande chance de l'obtenir: qu'il n'y ait aucun rapport entre le désordre social établi et l'harmonie à laquelle elle aspire - et surtout pas une relation génétique! Le monde mauvais n'aura pas le droit d'engendrer le monde meilleur. Faisons table rase et repartons de zéro!

Mince vélléité ! L'impuissance de l'utopie se trahit en tout domaine. Et déjà dans le fait que son nulle part ne lui sert souvent que d'alibi: «ne me persécutez-pas, je rêve seulement. Critiques sérieux, abstenez-vous! Je ne suis là pour personne».

Mais ni les amis ni les adversaires ne se laissent convaincre par cette palinodie. Ils n'accordent d'intérêt qu'à la démarche décidée de l'utopie, à ses descriptions précises, parfois aussi vivantes que celles d'un reportage. Elle restaure dans l'imaginaire les conditions de possibilité dont elle fait fi ordinairement. Elle se pourvoit d'une sorte de crédibilité.

Aussi comporte-t-elle le plus souvent, et paradoxalement, des indications de parcours, des itinéraires, des relais. Elle s'ingénie à baliser le chemin qui ne mène nulle part. Les dénigreurs de l'espace sont des maniaques du déménagement et de l'émigration!

L'utopie voudrait jouer de la rupture radicale, mais elle ne parvient pas à la penser, encore moins à la provoquer. Alors elle en rabat de ses prétentions premières. Elle se contentera d'un site écarté, de la distance à la place de l'absence. Elle recherchera les 
abbayes désaffectées, les vallées perdues, les continents lointains, et, surtout, les îles. A défaut, elle acceptera de camper sur une péninsule.

Que la mer sépare l'idéal du réel! Mais il faudrait d'abord, pour cela, que l'utopie convertisse les flots à cet usage. Un Dieu, dans sa sagesse, dit-on, a divisé la terre par des océans. Sans succès! $\mathrm{Ce}$ que les dieux proposent, les hommes en disposent. La mer ménage des médiations que l'utopiste lui-même ne dédaigne pas d'exploiter : il navigue, il colonise. Il n'oublie pas de donner de ses nouvelles, par la première frégate! pouvoir.

Le pouvoir de l'imagination n'est que l'imagination d'un

\section{La positivité de l'utopie}

Il n'est guère besoin de démontrer l'existence du lien de l'utopie au réel. Certes, à un ordre social mal élucidé, l'utopiste prétend substituer ses affabulations. Il annonce la meilleure des sociétés nouvelles sans trop se soucier d'évacuer la société périmée. Il forge donc des représentations fantastiques.

De ce fait, l'utopie fonctionne souvent comme idéologie sédative: pendant que l'on rêve, les choses suivent leur cours. Mais elle ne remplace pas toujours l'opium. Il lui arrive d'être tonique.

La fabulation qui l'engendre obéit elle-même, en effet, à des contraintes profondes : confie-moi tes rêves, je te conterai ta misère. Les hommes ne délirent que lorsque le monde a de la fièvre.

Les désirs qui suscitent l'utopie tourmentent vraiment l'humanité, ils naissent dans ce monde et de ce monde. L'utopiste les éprouve et les prélève, mais ne les crée pas. Leur satisfaction imaginative n'a rien de surnaturel. De quel autre monde pourraient donc surgir magiquement les prestiges d'un autre monde?

L'utopiste traduit à sa manière une carence et une insatisfaction qui restent insensibles, ou peu sensibles, aux esprits simplement positifs. Il voit, dans son époque, des aspects négatifs qui échappent au regard orienté du positiviste, et il fait appel à des 
suppléants fictifs. Les contradictions et les absurdités vécues, il les ressent et les juge comme un mal dépourvu de toute contrepartie, et, manichéen, il voudrait remplacer ce mal par un bien.

Aussi, même dans ses affirmations péremptoires et dans ses descriptions animées, quelque style qu'elle adopte, la pensée utopique ne perd-elle jamais son caractère essentiellement critique. Il ne faut pas s'y tromper: le tableau positif de ses visions n'est que l'habile traduction d'une négation. Elle dénonce, elle blâme, elle attaque, même si elle n'a pas l'air d'y toucher.

Toutefois, sa critique, ainsi enveloppée, se présente comme extérieure. Elle oppose abruptement l'autre au même. Elle souligne les différences, mais elle ne les aiguise pas jusqu'à la contradiction. Elle porte un jugement comme si elle était étrangère à la chose jugée, comme si elle n'en participait pas. Elle n'a que le pressentiment de la profondeur, et elle veut supprimer ce qui, sans qu'elle s'en aperçoive, est en voie de se supprimer soi-même. Elle creuse, à l'écart, la tombe des iniquités et elle n'entend pas s'élever de ces iniquités mêmes le chant joyeux des fossoyeurs.

Elle reste pourtant rattachée à ce qu'elle réprouve plus étroitement qu'elle ne le croit. On la tient en lisières. On lui prépare sa nourriture. C'est sur les chantiers publics qu'elle chaparde les matériaux de ses fables. Elle voudrait proposer l'inoui, elle n'agence souvent que des vieilleries. Parfois, elle met à nouveau en vitrine une marchandise vétuste, à peine époussetée.

En fait de surprise, la réalité dépasse toujours la fiction. C'est le passé qui s'offre d'abord à nous comme l'inconcevable, ou l'inimaginable, et nous nous habituons difficilement à lui. Aucun surréaliste n'aurait su inventer le culte d'Anubis ou le Droit romain.

Dans l'effort le plus extrême d'innovation, la chimère emprunte ses contours à la négation systématique de l'apparence présente: «De mort, il n'y en aura plus; de pleur, de cri, et de souffrance, il n'y en aura plus, car l'ancien monde s'en est allé ! »

Il ne s'en est pas allé. L'idéal fait partie du réel. Les utopistes expriment leur lieu, leur temps, leur milieu, et, souvent, ils les expriment mieux que ne le feraient des positivistes. On ne peut 
aucunement se fier aux analyses que ceux-ci ont données, à chaque époque, de la société dans laquelle ils vivaient. Au contraire, la plupart de ceux que l'on tenait en leur temps pour des utopistes ont fait preuve de perspicacité. Platon, dans sa République, a mieux saisi que beaucoup de ses contemporains la vraie nature de la Cité grecque: «La République de Platon, qui est l'image proverbiale d'un idéal vide, ne saisit essentiellement rien d'autre que la moralité grecque. Il a eu conscience d'un principe plus profond qui faisait brèche dans cette moralité, mais qui, à ce degré, ne pouvait être qu'une aspiration insatisfaite $»^{1}$.

La définition selon laquelle l'utopie serait «la doctrine fantastique d'un ordre social sans base réelle $\gg$ a donc besoin de compléments. L'utopie ne se soucie d'aucune base sociale, mais cela ne signifie pas qu'aucune base sociale ne se soucie d'elle. Il lui faut bien un support. L'aveuglement de l'utopiste ne saurait nous abuser: en fait, en étudiant une utopie ancienne nous dégageons assez aisément son enracinement social et politique, et, grâce à elle, nous parvenons à mieux connaître l'esprit du temps et la structure de la société.

Mais l'utopie trahit d'une autre manière sa positivité : quelle que soit sa nostalgie d'un ailleurs, elle ne renonce que bien rarement à intervenir bic et nunc. Elle le fait parfois en cachette, parfois sans vergogne. Déchirée, incertaine, elle appelle à l'évasion, mais, dans le même temps, elle incite à l'intervention. Elle veut être prise au sérieux au moins à moitié. La comparaison qu'elle institue entre un monde meilleur qu'elle fait miroiter, et un monde mauvais, qu'elle discrédite, réveille déjà le goût d'agir.

L'usage du mot utopique prend donc un sens polémique, tout à fait usuel. On est toujours l'utopiste de quelqu'un. Les positivistes ne s'emporteraient pas si hargneusement contre des contes de fées, anodins. Il existe bien des catégories de fantastique, et il est subversif de pousser certains hommes à rêver, quand il s'agit de certains rêves.

L'utopiste ne se confond pas avec n'importe quel fantaste. Pour bien marquer la différence qui le singularise, il entreprend

1. HEGEL, Philosopbie du Droit, Préface, Trad, Kaan, 1940, p. 29. 
parfois la réalisation de son projet nébuleux. Il boucle son havresac, et, suivi de la troupe des disciples, il embarque. Il exporte ses idées méprisées, mais il escompte bien les réimporter un jour glorieusement.

La déception, bien sûr, le guette. Il va inutilement chercher fortune idéologique en pays lointain: comme si la révolution pouvait consister en une promenade de quelques hommes sur la terre ! Comme si l'on plantait la justice sur une terre vierge à la manière d'un drapeau!

Il n'est que trop facile, et légitime, de reprocher à l'utopie sa naïveté mêlée de rouerie, et bien d'autres défauts.

Ceux qui émigrent découvrent d'autres paysages mais n'instaurent pas d'autres civilisations. Ou bien ils s'adaptent à celle qui les accueille, ou bien ils installent en pays désert le régime auquel ils ont voulu se dérober. On n'emporte pas la patrie à la semelle de ses souliers, mais on véhicule certainement un mode de production et des rapports sociaux déterminés dans sa boîte à outils. Ou bien on les maintiendra, sous un autre climat, ou bien on échouera. A peine arrivés sur la lune, les cosmonautes y déballent leurs superstitions. Un homme d'expérience nous en avertit : «On ne part pas!» (Rimbaud). De ce point de vue, il en va des sociétés comme des épistémies, leurs conjointes : pour elles, tout se joue toujours sur place.

L'utopie fait peu de cas de l'héritage et des moyens disponibles. Il s'agit de cécité plutôt que de négligence: elle ne voit pas correctement la structure sociale, et ne discerne pas les médiations qui s'activent en elle.

Alors, Fiat justitia! Un autre monde surgira! L'utopiste travaille dans l'inconditionné. Il n'existe pas, dans ce qui se dissout et meurt, les forces naissantes qui inaugureront une nouvelle vie. Il court-circuite les médiations. Reconnaissons-le : l'époque où fleurit l'utopie est en général celle où ces médiations ne se manifestent, sporadiquement, que comme des esquisses hésitantes, à peine discernables.

La portée politique d'une utopie ne saurait donc être appréciée sans une référence précise à son lieu historique. Selon les 
dates et les circonstances, le même système utopique peut participer d'une idéologie révolutionnaire, ou réactionnaire, ou quiétiste. La plupart du temps, il s'en tient au Sollen dont Hegel dénonçait la vacuité et la vanité.

La critique utopiste ne garde de sérieux qu'autant qu'elle n'est pas surclassée par une pensée globale du processus historique. C'est un pressentiment que la science et l'action discréditent en fondant une prévision et un plan.

Cette déchéance fatale reclasse l'utopie mais ne l'oblitère pas. Sans un tel préalable, la science ne naîtrait jamais. Marx a emprunté littéralement les mots d'ordre ultimes de son programme à des utopistes qu'il vénérait comme les «patriarches du socialisme ». Cela ne l'empêchait pas de combattre l'utopie en tant que telle, et, surtout, d'accabler, parfois avec violence, ses survivances anachroniques.

\section{L'utopie de la positivité}

Toutefois, la condamnation scientifique de l'utopie n'entraîne pas du tout l'absolution de ses adversaires! On l'oublie trop souvent, et cet oubli mériterait une analyse.

Pourquoi le jugement, même rétrospectif, reste-t-il partial en cette affaire? Il favorise injustement le positivisme.

Or celui-ci se révèle, à l'usage, tout aussi fantastique que l'utopisme. Thomas More, le téméraire, divaguait-il donc plus que ses contemporains timorés ou sanguinaires? Ceux-ci croyaient s'en tenir à la réalité. Mais leur bon sens les mystifiait cruellement, et, pour connaître leur époque, maintenant, nous ne recourons guère à leur témoignage. L'ordre social dont ils se satisfaisaient, ils ne le saisissaient que dans des représentations illusoires, dans une fausse conscience. De plus, ils en admettaient à la fois la cohérence indissoluble et l'éternité, ils ne prévoyaient aucunement les crises qui allaient bientôt le secouer et l'abattre, ils n'imaginaient même pas qu'autre chose fût possible. Ils pratiquaient le culte du fait accompli, mais ne constataient pas leur propre ignorance. Ils ne comprenaient rien, et leur nom n'échappe à l'oubli que parce que More les a affrontés. 
Les adversaires de Cabet s'estimaient plus « réalistes » que lui, et ils se moquaient du pauvre illuminé. Mais n'insulte pas qui veut! Quid rides, fabula de te narratur! Toute leur pensée se déployait dans le fantastique, et même dans le fantasmagorique, si étrangère au réel, si erronée qu'elle a perdu tout intérêt. Se souvient-on des «idées» régnantes de Charles $\mathrm{X}$ ou de LouisPhilippe? Autant en emporte la moindre brise de l'histoire!

Qui donc errait le plus, de Fourier ou des esprits soi-disant rassis qui le narguaient? On lui reprochait de demander l'impossible. Mais ses critiques ne pensaient eux-mêmes que dans l'impossible. Quelle signification, quelle valeur ont conservées les «théories» libérales au nom desquelles on le réprouvait, et qui ont toutes fait fiasco? Qui donc considère encore JeanBaptiste Say comme un penseur qui, après les divagations utopistes de sa jeunesse, aurait reconnu les choses telles qu'elles étaient, alors que Fourrier, de son côté, aurait déliré sans fin ?

Dans un passage saisissant du Capital, Marx dénonce la mystification dont sont victimes, à son avis, les économistes positivistes, des contemporains de Cabet ou de Fournier: ce qui se présente à leur conscience obnubilée, c'est «le monde enchanté et inversé, le monde à l'envers où Monsieur le Capital et Madame la Terre $(\ldots)$ dansent leur ronde fantomatique ${ }^{1}$.

Les positivistes s'abandonnent sans inquiétude à leurs lubies. Si l'on consent à appeler utopie «un genre littéraire qui édifie des sociétés imaginaires », alors presque toute la littérature sociale et politique, du moins jusqu'à la moitié du XXème siècle, relève de ce genre. De nos jours, certains s'enhardissent jusqu'à prétendre que Marx lui-même n'aurait pas réussi à s'en dégager tout à fait!...

Le bon sens privilégie l'aspect positif de la réalité, les fonctionnements corrects que l'on y peut isoler, et, plus généralement, la pellicule des apparences immédiates. Il ne soupçonne pas qu'il y a un revers de ces apparences, que le positif implique le négatif, que les choses ont une profondeur. Il s'endort sur la surface plate du monde, et, pour des raisons que l'on pourrait dévoiler, l'en soi qui se dissimule sous l'apparence lui reste inconnu. Son regard ne se dirige que vers les faits, vers ce qui est déjà 
fait, insensible à ce qui aspire à être, à ce qui reste à faire, à ce qui point.

La complaisance au fantastique ne caractérise donc pas l'utopisme plus que le positivisme. L'un n'est pas plus libre que l'autre.

\section{La même galère}

Les hommes d'une même époque soutienne dans une même citadelle épistémologique le siège de leurs souvenirs. Avec le temps ils deviennent les prisonniers de leurs propres remparts.

Les pensées d'un utopiste et d'un positiviste contrastent beaucoup moins, s'ils sont contemporains, que les types de pensée de deux époques différentes. Il faut bien que les utopistes et les positivistes pensent la même chose, quoique de manière différente, pour s'y intéresser ensemble. Ils mettent en commun le champ de bataille et l'enjeu. Ils se fournissent chez le même armurier.

Ils éprouvent sans doute la même perplexité devant le fétichisme primitif, mais ils se comprennent mutuellement assez pour se combattre. Une sorte de complémentarité les relie. Le sage Utopus ne feuillette pas, sur son île, un autre dictionnaire que celui de More et d'Erasme.

Renonçons donc à établir une coupure absolue entre l'utopie et la positivité. Leur différence s'efface, après quelques siècles. Quand nous les contemplons du haut de notre épistémie nouvelle, elles paraissent également aberrantes.

Nous ne pouvons toutefois les confondre entièrement. Qu'est-ce donc qui les distingue et surtout, qu'est-ce qui les distinguait en leur temps?

C'est comme si, à la fois inséparables et opposées, elles se partageaient une tâche trop lourde pour un seul ouvrier. L'utopiste et le positiviste profitent d'une division du travail, et en même temps ils en pâtissent. L'un laboure le positif, à un bout du champ et l'autre creuse le négatif, à l'autre bout, tous deux oublieux de l'unité des deux pôles, tous deux asservis à leur besogne parcellaire, ignorants de leurs propres limites, captifs et indispensables : 


\section{«La vie}

\section{Le réel et l'imaginaire}

Sont ses deux mains et ses deux yeux; ${ }^{2}$

Un jour peut-être ces deux yeux se fondront-ils en un seul regard. Mais, en attendant qu'une synthèse restaure cette unité première en la transfigurant dans un surrationalisme auquel avait songé Bachelard, chacun continue de porter des jugements abstraits et unilatéraux.

L'utopiste, vivant dans l'ombre, ne la reconnaît pas comme le double négatif de la clarté. Le positiviste, ne tolérant que la lumière, perd la moitié de soi-même.

Tous deux omettent l'essentiel, le dynamisme de l'essence, la germination, la positivité du négatif que le monde engendre et qui, à la longue, le transforme. Ils méptisent les germes, réconfortants ou désespérants. "L'impatience demande l'impossible : atteindre le but sans les moyens $»$, dit $\mathrm{Hegel}^{3}$. On pourrait ajouter: «La paresse demande aussi l'impossible: qu'il n'y ait pas de but ni de moyens».

L'utopiste bricole un paradis, plus ou moins séduisant, affranchi de toute oppression et de toute humiliation. Mais il n'envisage pas que la condition humaine puisse être modifiée par les hommes qui la subissent. Alors, il leur octroie souverainement la liberté et la dignité, le bonheur. Une telle dotation gratuite contient pourtant la négation même de la liberté : celle-ci n'appartient qu'à ceux qui la gagnent. L'utopiste ne récupère rien dans le monde en perdition: en fin de compte ce monde le récupérera, comme il récupérera aussi le positiviste.

D'autres signes révèlent la parenté de ces deux rivaux, malgré leur opposition, ou plutôt à cause d'elle. D'abord, quelle que soit leur affectation de dégoût réciproque, l'utopisme et le positivisme se mêlent dans les oeuvres d'un même auteur de la manière la plus réjouissante. Par une grâce exceptionnelle ils réussissent même, épisodiquement, de somptueuses synthèses : une coïncidence momentanée avec le processus réel.

2. Paul ELUARD : Le Château des pauvres.

3. Préface à la Pbénoménologie de l'Esprit, Paris, Aubier-Montaigne 1966 p. 73. 
Mais même lorsqu'ils prennent leurs distances, ce n'est que synchroniquement. Diachroniquement, ils se renversent communément l'un dans l'autre, laissant alors tomber les masques.

On l'a trop souvent noté: "Les utopies ne sont que des vérités prématurées » (Lamartine). Les navettes courent maintenant toutes seules, les poules ont des dents. Prenons acte de la réalisation de mainte utopie, même si elle s'accompagne de surplus imprévus et indésirables.

Par contre, on fait généralement silence sur ce qui est le plus intéressant: les théories "positives», et en particulier les acquis scientifiques, prennent, avec le temps, une allure tout à fait fantastique. La pensée conservatrice, presque toujours dominante, préfère ne pas se souvenir de ce destin, qui préfigure son propre avenir.

A bien des égards, les hommes, jusqu'à nos jours, ont vécu dans la réalité et pensé dans l'illusion, les pieds dans la boue et la tête dans les nuages. Peut-être cette mystification les a-t-elle aidés à vivre. Auraient-ils supporté, sans elle, leur condition à la fois odieuse et nécessaire?

Nous ne retenons pour vraie, ou pour acceptable, aucune des conceptions globales de l'existence qui se sont succédées jusqu’à nous au long des millénaires. A peine parvenons-nous, au prix d'un long effort, à les repérer, et à les comprendre partiellement: "Nous ne pouvons tout de même pas partager les sentiments des Grecs lorsqu'ils se prosternent devant Jupiter ou Minerve! Pas plus que nous ne pouvons comprendre les sentiments d'un chien ${ }^{4}$. En vieillissant l'essentiel perd son sens.

Un projet devient utopique quand la nécessité l'abandonne, et elle l'abandonne fatalement quand son heure est venue. La société réelle rajuste alors ses vêtements, et elle range dans des musées les plus beaux des rêves surannés. Si ce spectacle réchauffe quelques nostalgies, il ne suscite plus de drames.

4. HEGEL, Lefons sur la pbilosopbie de l'bistoire, Introduction in WERKE (éd. Hoffmeister), Hambourg, Meiner, 1955, pp. 13-14. 


\section{Le lieu de l'utopie}

Les changements se produisent donc sur place. Dans quel «ailleurs» pourrait s'effectuer la mutation du positif en négatif, ou la révolution qui bouleverse un ordre social?

Or l'utopiste n'espère pas qu'un autre monde survienne dans ce monde-ci. Il ne s'y attend pas empiriquement et il ne conjecture pas méthodologiquement cette éventualité. En cela il ne se montre pas meilleur dialecticien que le positiviste. La dialectique lui rendrait insupportable l'idée d'une rupture radicale, elle l'inviterait heuristiquement à rechercher des médiations dans la réalité, et, si elles n'y sont pas visibles, à les y présupposer : elles doivent y exercer leur action, même si elles se cachent. Il n'y a rien qui ne contienne en soi son contradictoire, avec lequel il forme une unité. Mais l'utopiste cède à la tentation du dualisme, ou même du pluralisme.

Le changement ne se réduit pas à une substitution : un clou chasse l'autre, une diapositive s'éclipse devant la suivante. Seuls des fragments intellectuellement ou manuellement manipulés peuvent donner l'illusion d'une substitution abrupte - à condition d'oublier l'intelligence et la main manipulatrices. Sans médiations, la catastrophe serait un miracle. La stroboscopie restitue l'image changeante de l'enchaînement vivant.

La société, comme connexion de rapports sociaux, ne déménage jamais. Elle se renverse en son contraire, spontanément, ou avec l'aide de ses membres. Elle ne change pas de lieu, mais change en son lieu. Le concept de substitution ne subsume que très imparfaitement un événement de ce genre.

En 1789, la structure politique française n'a été ni transportée, ni déplacée, mais renversée à Paris même. Le révolutionnaire n'émigre pas. Il reste chez lui. La première condition de la liberté, c'est de ne pas fuir.

On ne peut pas mettre le social à la porte de la société. On ne peut mobiliser une force qui ne soit déjà engagée dans le jeu des forces. Le poète nous avertit : «Il y a un autre monde et il est dans celui-ci » (Eluard). 
Or la pratique de l'utopiste implique que la pensée ne dépend d'aucune condition. Cette autonomie spirituelle, voilà la forteresse factice qui protège sa hardiesse abusive et qui, simultanément, lui impose ses limites étroites: "Quelle est donc la cause qui détermine sa volonté ? c'est son jugement. Et quelle est la cause qui détermine son jugement? c'est sa faculté intelligente, c'est sa puissance de juger : la cause déterminante est en lui-même. Passé cela, je n'entends plus rien ${ }^{5}$.

Le véritable lieu de l'utopie consiste en une croyance qu'elle partage avec la plupart de ses adversaires: on se constituerait soimême comme utopiste ou comme positiviste, comme visionnaire ou comme clairvoyant, par un libre choix. «Passé cela», l'utopiste n'entend plus rien. Que le contenu de l'utopie ait des sources d'inspiration, qu'il dépende d'expériences effectives, passe encore! Mais l'option en sa faveur reste libre, l'utopiste ne se considère pas comme un produit du monde qu'il réprouve. Il prend une voix d'outre-tombe. Il investit précisément toute sa liberté dans une négation radicale de ce monde, auquel il refuse l'allégeance! Il n'est que ce refus. La liberté de l'utopie suppose l'utopie de la liberté. Le lieu décisif n'est donc pas celui où l'utopiste situe avec mauvaise foi sa société idéale. Mais c'est celui qu'il occupe luimême, sans le savoir, et sans même se poser de question à son sujet, tant il est convaincu d'être un ou-topos! Ce qu'il y a de plus utopique, dans l'utopie, c'est l'utopiste.

Voilà l'utopie fondamentale, et très répandue : le rejet d'une géographie de la pensée. Pour se justifier, ce rejet doit recourir, à son tour, à toutes les manigances, et se couvrir de tous les masques. Il lui faut de la place pour se dire, et il abuse des topologies géométriques, rhétoriques, triviales. Les lieux-communs s'épuisent à situer le non-spatial, l'au-delà, le céleste, le transcendant...

La théorie serait en rupture radicale avec le monde. Epistémè enverrait promener Cosmos. Comme dit l'autre, d'une manière si touchante: «Fuyons vers notre chère patrie»!

5. J.J. ROUSSEAU, L'Émile, Livre IV - (Oeuvres complètes, éd. Gallimard, IV, p. 586). 
Mais il est plus facile de le dire que de le faire, s'il est toutefois possible de le dire. La promenade et la fuite ne peuvent se passer d'un chemin. L'utopisme épistémique effectue les mêmes replis stratégiques que l'utopisme social. Par des itinéraires embrouillés, ils se rencontrent sur la même île, piteusement, comme s'ils s'y étaient donné rendez-vous. Mais c'est l'île qu'ils croyaient n'avoir jamais quittée, persuadés qu'ils y étaient nés : "L'esprit est une île à laquelle quels que soient les détours empruntés, on ne pourra parvenir, en partant de la matière, que par un bond ${ }^{6}$.

Après un tel isolement, il semble bien difficile de rejoindre raisonnablement la terre ferme. Toutes les communications sont coupées : "L'idée des choses corporelles ne peut d'elle-même atteindre un objet qui, s'il existe, est au-delà d'elle $\gg . .{ }^{7}$

On ne s'y retrouve plus.

Mais n'était-ce pas le but de l'opération, secret ou inconscient?

L'illusoire absence de lieu de la pensée autorise en effet toutes les autres illusions, leur confère une sorte de statut légitime. Le fantastique a le champ libre, aussi bien dans la représentation «positive» que dans la prospective utopique. Pourvu de cette caution théorique, il se lance même à l'assaut du passé, commettant, cette fois, une fraude. Mécontent des témoignages historiques, Lachelier ne craignait pas de les récuser, au nom de la liberté de l'esprit, et de les remplacer par une utopie rétrospective : «Et quand cela serait arrivé - écrivait-il à un ami - il faudrait dire, plus que jamais, que cela n'est pas arrivé, que l'histoire est une illusion, et le passé une projection, et qu'il n'y a de vrai que l'idéal et l'absolu; là est peut-être la solution de la question du miracle; c'est la légende qui est vraie et l'bistoire qui est fausse ${ }^{8}$.

More et Cabet n'en prenaient pas plus à leur aise! Leurs utopies ne rendaient pas un aussi grand hommage à la résistance du réel : "Et quand bien même cela serait arrivé !...»

6. SCHELIING : Système de lidéalisme transcendantal, C.S.W., III, p. $429-$ éd. Schröter, II, p. 429.

7. V. DELBOS, La Pbilosopbie française, p. 35.

8. Letire à Boutroux du 21 Janvier 1876 (Correspondance, pp. 113-114). 
Mais comment se laisserait-on prendre au piège des mots, une fois qu'il est éventé ? Vient un temps où ces supercheries n'en imposent plus, et où l'on remise tout cet attirail de sorcellerie.

On rompt alors avec l'idéologie de la rupture. On délaisse le mythe de l'ou-topos.

Cette libération ne s'accomplit pas sans douleur. Il n'est pas toujours si agréable de rentrer chez soi, où le travail attend, lorsqu'on a pris l'habitude de flâner dans le merveilleux.

Cet effort d'émancipation, aux yeux de beaucoup de nos contemporains, c'est bien «le comble »! Ce n'est pas leur manière habituelle de s'orienter dans la pensée. Ils empruntent plutôt la direction opposée. Ils comprennent la liberté essentiellement comme pouvoir de nier - peut-être parce qu'ils sont séparés, spécialisés, mutilés, isolés. Ils résistent à l'amalgame, ne se laissent pas réduire à de simples modes.

Ils font tout pour bondir hors de l'espace, par-dessus l'espace, hors du temps, par-dessus le temps : s'ils réussissent, la liberté les récompensera, elle qui n'a pas de séjour. Il s'agit là d'une conversion qui leur paraît pénible, d'un retournement de toute leur âme vers l'idéal. Ils n'osent croire qu'ils l'achèveront jamais complètement. Ils l'entreprennent, parfois sans espérer, et l'entreprise déjà les fascine. Et puis l'enjeu en vaut la peine! Et voilà qu'on vient leur proposer une sorte de saut à rebours! Cessera-. t-on de les faire virevolter? Déjà la simple idée de renoncer à l'autonomie leur donne le vertige.

Mais il leur faut bien alors reconnaître que ce qu'ils dé. crivaient comme trop facile, une attitude naïve et niaise, spontanément séduisante, se révèle, à l'épreuve, comme le plus difficile, peut-être l'impossible.

L'utopisme est un mol oreiller. Pour qui s'y repose, la Terre des hommes paraît infiniment éloignée: Hic Rhodus, bic salta!

Quel n'est pas le trouble de l'utopiste quand il se voit convié à risquer dans l'espace ce bond terrible que Hegel lui reprochait d'effectuer hors du temps: «En ce qui concerne l'individu, chacun 
est fils de son temps; de même aussi la philosophie, elle résume son temps dans la pensée. Il est aussi fou de s'imaginer qu'une philosophie quelconque dépassera le monde contemporain que de croire qu'un individu sautera au-dessus de son temps, franchira le Rbodus ${ }^{9}$.

Sautera, ne sautera pas? Hegel a raison : il n'y a pas à revenir, on n'est jamais parti. Il faut simplement disperser quelques brouillards. Lorsque la brume se lève, au lieu d'une île, l'esprit découvre un monde sans rivages.

Ne nous vantez plus vos attolls dérisoires et votre arrièremonde de pacotille! L'exotisme ne fait plus recette. L'utopie se meurt, elle s'engloutit dans les entrailles du monde réel. C'est lui que, maintenant, nous sentons trembler dans ses profondeurs. Sous l'apparence tranquille des choses, nous identifions le négatif qui le ronge. Il est la liberté et la vie, et nous y trouvons notre liberté et notre vie. Etre libre, c'est Iui appartenir.

Université de Poitiers

9. Philosopbie du Droit, Préface, Trad. Kaan, Paris, 1940, p. 31. 